\title{
Case Report: - A Case of Pulmonary Aspergilloma
}

\author{
Yasser Mubarak, MD. \\ Assistant Professor of Cardiothoracic Surgery, Minia University, Egypt.
}

\begin{abstract}
Virchowdescribed the pathology of human aspergillosisin 1856. Hinson and colleagues classified pulmonary aspergillosis into allergic, invasive, and saprophytic infections. Saprophytic type colonized in pre-existing lung cavity and produceda fungus ball, or aspergilloma. (4)
\end{abstract}

Pulmonary aspergilloma (fungus ball or Mycetoma) is rare pulmonary infectious disease. Its clinical manifestation includes; chronic cough, fever, dyspnea, chest pain, hemoptysis and others, but it may be asymptomatic. (1)It is a potentially life-threatening disease that is difficult to treat without surgical intervention, which is the treatment of choicein selected cases. It does not respond to antifungal agents alone.(5)In cases of high surgical morbidity and mortality, alternative the rapiesare needed.(3)Bronchial artery embolization can be used to treat massive hemoptysis but is rarely completely effective due to the existence of massive collateral blood vessels.(5)

Keywords:- Aspergilloma, Fungus ball, Cavitary.

\section{INTRODUCTION}

Pulmonary aspergilloma is fungal infection by Aspergillus Fumigatus. (1)It may be asymptomatic inhealthy hosts, chronic necrotizing aspergillosis in chronic lung disease, invasive aspergillosis in immune compromised hosts and allergic broncho-pulmonary aspergillosis in asthmatics.(2)

Chest radiology may provide characteristic pictures of this disease, but pathological examinationis needed forsure definitive diagnosis. Current treatments include antifungal regimens and surgical resection. (1)Pulmonary aspergilloma is the only surgicallyrelevant manifestation of Aspergillus infection. (2)It produces significant morbidity and requires early surgical intervention. (5)

\section{CASE REPORT}

A 72-year-old woman with $\boldsymbol{C O P D}$ (chronic obstructive pulmonary disease) was admitted to Minia University Cardiothoracic Hospital after suffering from intermittent moderate hemoptysis(150-300 ml/24 hr.), chronic cough, and dyspnea. No history of TB, cardiac disease, anticoagulant taken, bleeding tendency, or malignancy. At the thoracic surgery department, chest radiograph was done. Diagnosis of aspergilloma was made primarily on the radiological examination showing "fungus ball" on plain chest X-ray and demonstration of pulmonary cavity $(6 \mathrm{~cm} \mathrm{X}$
$8 \mathrm{~cm}$ ) with free ball lesion on CT and revealed a large cyst of right upper lobe $(\boldsymbol{R} \boldsymbol{U} \boldsymbol{L})$ of the lung. Figure(1-2-3)All vital signs were within normal range. Laboratory studies revealed hemoglobin level of $8 \mathrm{gm}$. and corrected by blood transfusion. Liver function and platelets count were normal. Sputum acid-fast stain and cytology were negative to TB and malignancy. Pulmonary function test was revealed obstructive pattern and unfit for lobotomy. Once date of admission, antifungal medication was taken. The patient was prepared for surgery. Just before thoracotomy, bronchoscopy revealed no endobronchial lesion or hemorrhage. The patient was operated under general anesthesia, with double lumen endotracheal tubes for adequate isolation and selective ventilation, through standard postero-lateral thoracotomy Wedge resection of RUL cyst was performed and specimen was sent to histopathological examination and fungal culture. Figure (4) Post-operative course passed smoothly without air leak in ICT (inter costal chest tube), hemoptysis, fever, or wound infection. The patient was discharged on $5^{\text {th }}$ POD (postoperative day) with clean wound. The pathological examination of the lung biopsy revealed characteristic findings of pulmonary a spergilloma cavity filled fungal ball that was composed of numerous acute angled branching septated hyphae. The patient completed antifungal course medication with normal chest CT (Computed Tomography) 3 months later.

\section{DISCUSSION}

Pulmonary aspergilloma is a chronic fungal infection withmortality incidence from massive hemoptysis ranging between (2\% -14 \%).Aspergillusmay growon the preexisting lung cavity formed secondary to a variety of causes, such as TB, sarcoidosis, bronchiectasis, bullae, and malignancy. (3)

Aspergilloma does not respond to antifungal regimen, as the drugs rarely achieveminimal concentration within lung cavity. (3)Pulmonary aspergilloma can cause sudden and massive hemoptysis. The incidence of hemoptysis inpulmonary aspergilloma has ranged from 50\%-83\% and is severe or recurrent in $10 \%$.(2)Different mechanisms for hemoptysis have been proposed included erosion of the vascularcyst wall, and production of endotoxin. The first hemoptysis may be massive and fatal due to unclear cause. The size of the aspergillomas bore no relation to the severity of hemoptysis. (4) In our case report, the patient was manifested by recurrent moderate hemoptysis. 
Surgery is the treatment of choice but may be impossible due to poor pulmonary function test. It has a mortality incidence between $7 \%-23 \%$.( 3) Pulmonary function test in our case was bad but provide possibility to wedge resection.

Aspergilloma was noted that upper lobes of lungs were the favoredsite of aspergillomas.(2) The upper lobes are the predominant sites of aspergillomas.(4)Also, mycetoma presented in RUL in our case.

Successful management of pulmonary aspergilloma needsboth surgery and perioperative drug therapy. Surgery offers symptomatic control and alsoimprovessurvival.(2)

Prophylactic resection of pulmonary aspergilloma was recommended because of the risk of massive hemoptysis and death. (4)A marked decline in mortality and morbidity due to surgery has led to its greater acceptance in recent years. (2)

Otherauthors recommended surgical resection only after hemoptysis has occurred. Some of patients have advanced chronic lungdisease to a degree that eliminates the surgical resection. So, surgical resection should be avoided because of the high incidence of postoperative complications. (4)

Simple aspergilloma is amenable to surgery and has low operative risk, if good pulmonary reserve is present, prior to surgery, but complex aspergilloma results in maximum post-surgical complications and even death. Preoperative pulmonary function test can indicate immediate outcome after surgery and on long term. (5)

Surgery is indicated in all cases, by some, to avoid life threatening hemoptysis, while others prefer surgery only in symptomatic cases, due to high surgical morbidity and mortality (0-4.3\%).(5)

The main goal of the surgical procedure is to resectcavitation near the pulmonary vessels and localized pulmonary diseaseto prevent fatalhemoptysis although limiting the parenchymal resectionas much as possible not to impair lung function.(4)

\section{CONCLUSION}

Pulmonary aspergilloma is a rare pulmonary infectious disease. Early diagnosis is based on radiological findings which may provide some clues. Surgical resection of symptomatic cases is important to prevent recurrence and massive hemoptysis. Surgical option is safe in selected case with appropriate pulmonary function. Immediate and late post-operative course is well.

\section{REFERENCES}

[1]. Chen W, Ming L, Ruey H, HuiC, Heng S.A case of pulmonary aspergilloma and actinomycosis.J Med Microbiol.2011; 60: 543-46.

[2]. Himanshu P, Dewan R, Singh I, Gill S, and VaddadiS. Surgical Treatment of Pulmonary Aspergilloma: A Seriesof 72 Cases.Indian J Chest Dis Allied Sci. 2007; 49: 23-27.

[3]. David R, Alain T, Paul M, Alex C, Elaine D, Gary A.Bronchoscopic Removal of a Large Intra-cavitary Pulmonary Aspergilloma.Chest. 2013; 143(1): 238 41.

[4]. Gerard B, Massimo M, Alain C, et al. Surgical treatment ofpulmonaryaspergilloma: current outcome. $J$ Thorac Cardiovasc Surg. 2000; 119:906-12.

[5]. Mayuri A, Anand P, Ashok T. Case Report: Chronic cavitatory aspergilloma in an old emaciated patient with review of three cases.Int Surg J. 2014;1(1):45-49.

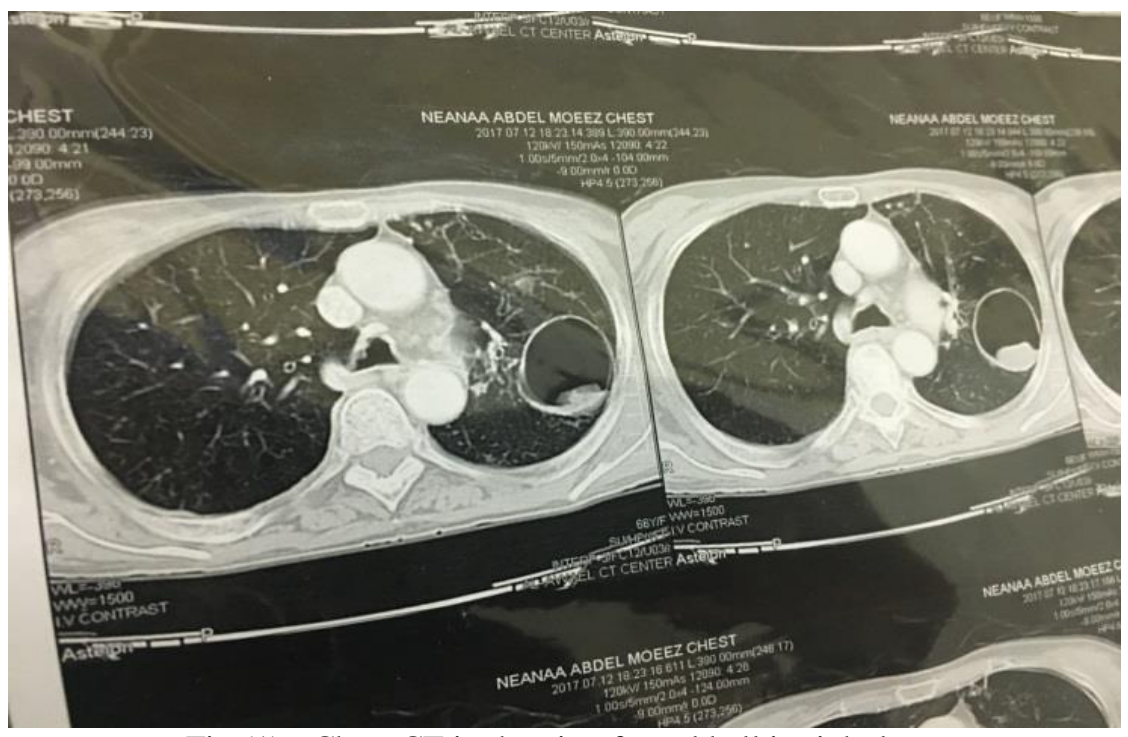

Fig (1):- Chest CT is showing fungal ball in right lung 


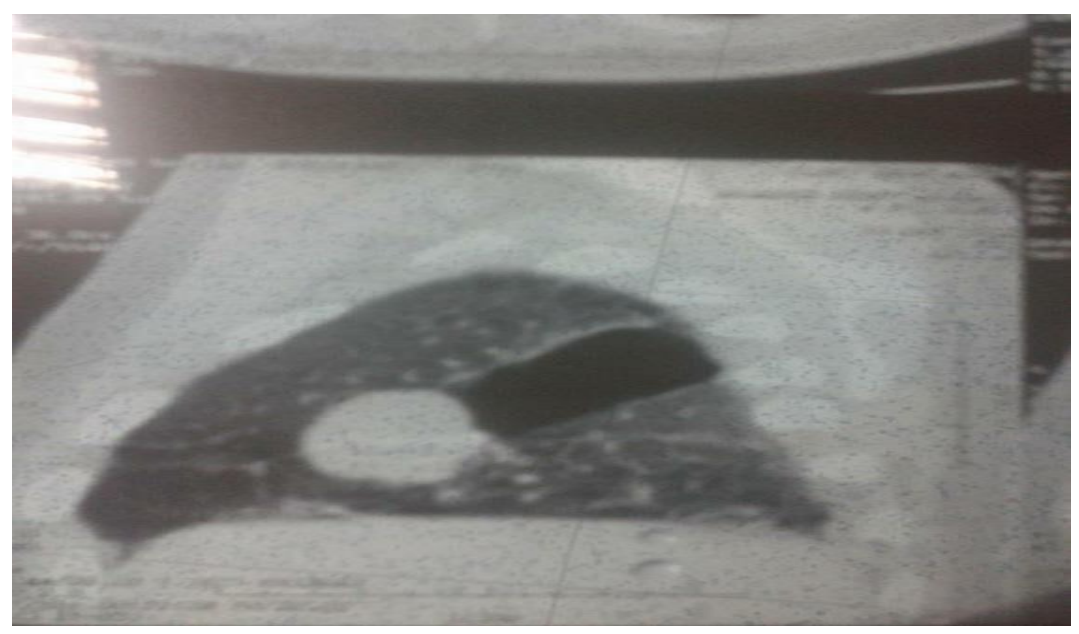

Fig (2):- Chest CT is showing fungal ball in previous lung cyst

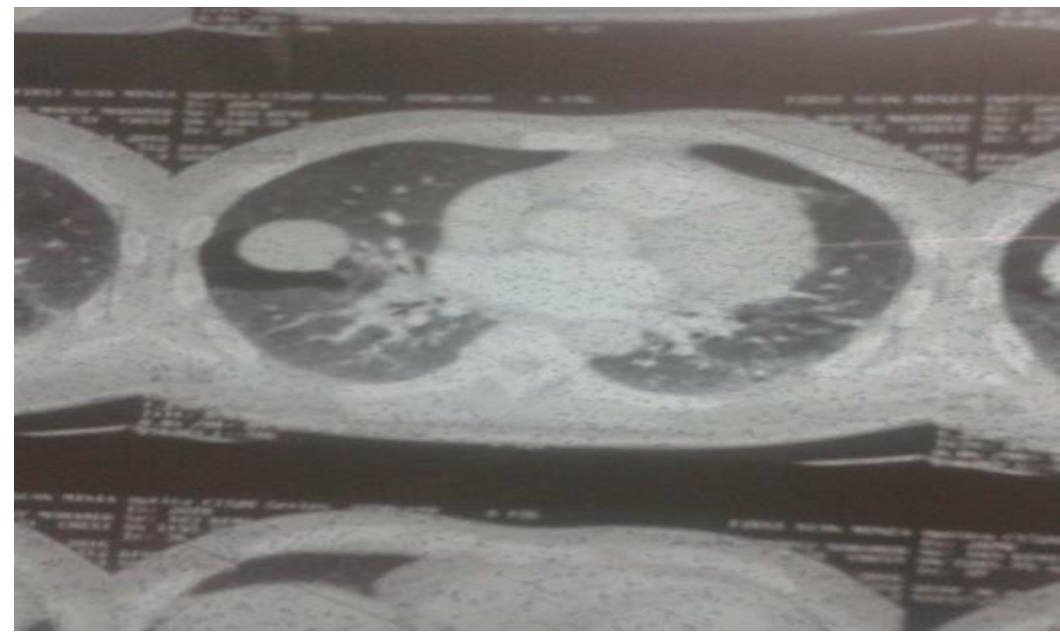

Fig (3):- Chest CT is showing fungal ball in left lung with interstitial lung fibrosis
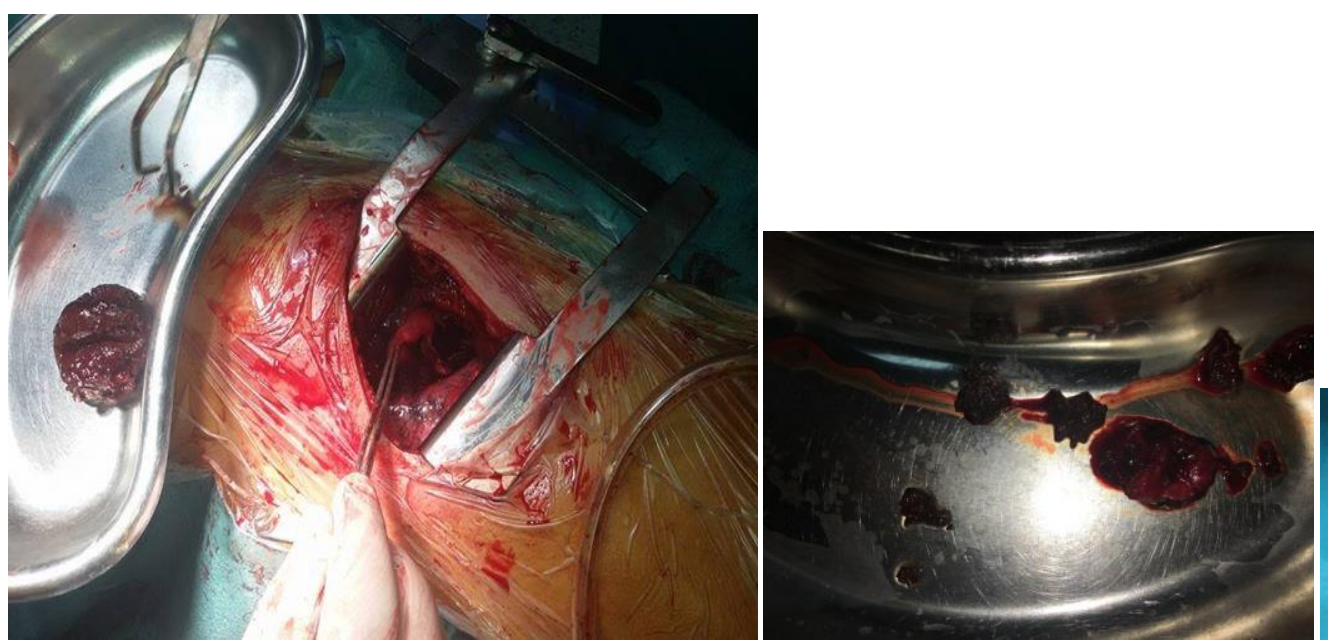

Fig (4):-That is showing thoracotomy with extraction of fungal ball 